\title{
Effect of Cold Rolling on the Structure and Hydrogen Properties of AZ91 and AM60D
}

\section{Mg Alloys Subjected to ECAP.}

Alberto Moreira Jorge Jr. ${ }^{1,2,3 *}$,Egor Prokofiev ${ }^{1,4}$, Maria Regina Martins Triques ${ }^{1}$, Virginie Roche ${ }^{2}$, Walter José Botta ${ }^{1,2}$, Claudio Shyinti Kiminami ${ }^{1}$, George I, Raab ${ }^{4}$, Ruslan Z. Valiev ${ }^{4}$, Terence G. Langdon ${ }^{5 *}$

${ }^{1}$ Departament of Materials Engineering, Federal University of São Carlos, São Carlos 13565905, SP, Brazil

${ }^{2}$ LEPMI Laboratory CNRS, UMR 5266 INPG - UJF, Grenoble, BP75, 38402 St-Martin d'Hères, France

${ }^{3}$ SIMAP laboratory CNRS, CNRS UMR 5266.INPG - UJF, Grenoble, BP75 - 38402 St-Martin d'Hères, France

${ }^{4}$ Institute of Physics of Advanced Materials, Ufa State Aviation Technical University, 12 K. Marx str., Ufa 450000, Russian Federation

${ }^{5}$ Materials Research Group, Faculty of Engineering and the Environment, University of Southampton, Southampton SO17 1BJ, UK

\section{ABSTRACT}

It is attainable obtaining an appreciable amelioration of the hydrogen storage ability in magnesium by settling on a suitable sequence of mechanical processing. It is plausible to predict that a reduction in grain size into nanocrystalline dimensions may predispose to a substantial improvement in the diffusion and thermo-dynamical properties in magnesium hydride. This may be realized by Severe Plastic Deformation (SPD). The use of cold rolling after ECAP, a kind of SPD, considerably increases the preferential texture for hydrogenation. In this research, the association of ECAP and cold rolling was used to process commercial AM60D and AZ91 extruded alloys in order to evaluate their use as hydrogen storage materials. The ECAP was conducted under distinct conditions of numbers of passes in order to attain different grain sizes. After ECAP, both materials were cold rolled with the same 
number of passes for comparison of hydrogenation absorption. Hydriding properties are enhanced by (0001) texture, which improved kinetics mainly in the initial stages of hydrogenation and was effective even with unfavorable grain size. However, the presence of precipitates limits the storing capacity. Finally, it was demonstrated that optimum sorption properties may be acquired with the synergy between fine grains and the best texture.

Keywords: Equal-channel angular pressing; Hydrogen storage; Magnesium alloys; Severeplastic deformation; Ultrafine grains

*Corresponding authors: A.M.J.J - moreira@dema.ufscar.br, T.G.L - langdon@usc.edu 


\section{Introduction}

It is now well established that increasing global warming due to gaseous greenhouse emissions is seriously affecting the environmental and political climates. Thus, it is becoming clear that there is a very significant demand for alternative fuels. Under these conditions, hydrogen arises as an option of clean energy in which water is the sole sub-product after burning. Despite this attraction, there are several shortcomings in the current technology for hydrogen storage and the solutions to these problems present serious challenges in terms of safety, energy density, efficiency and cost.

Considering their volumetric capacity and safety, metal hydrides appear to be adequate and magnesium is a strong candidate for a hydrogen storage system because it has a high hydrogen storage capacity (7.6 wt.\% hydrogen), reasonable cost and, in the form of bulk metal, a good safety record $[1,2]$. To date, a number of hydrides based on magnesium or intermetallic compounds [3] have been fabricated. Nevertheless, magnesium metal possesses unsatisfactory hydrogenation properties wherein the kinetics of hydrogenationdehydrogenation are remarkably slow with the reactions occurring only at very high temperatures $[4,5]$. These poor kinetics are mainly due to the low diffusivity of hydrogen in the magnesium hydride $[4,5]$ and in practice the presence of surface oxide layers may delay or even hamper hydrogen penetration into the bulk $[6,7]$.

Several efforts have been dedicated to improving the kinetics through, for example, surface oxidation control $[8,9]$, surface modification [10], particle size reduction [4-7, 11-15] or the addition of different catalysts [7-9, 16-22]. A recent review summarized the use of metal hydrides material for solid-state hydrogen storage applications [23].

It is reasonable to anticipate that a reduction in grain size to the nanometer range may substantially improve the diffusion and thermodynamic sorption properties in magnesium and this may be achieved most readily through the application of severe plastic deformation 
(SPD) [24-30] since SPD processing is capable of producing Ultra Fine Grained (UFG) or nanocrystalline materials from conventional coarse-grained bulk metals. This high microstructural refinement is achieved by deformation under a high hydrostatic pressure and at relatively low deformation temperatures [31-33].

The most common and efficient type of SPD is equal-channel angular pressing (ECAP) [32] in which numerous light metal alloys have been processed to achieve exceptional grain refinement. Furthermore, several studies report the use of ECAP in Mg and its alloys for the improvement of their sorption properties and their structural cycling stability where these superior properties are attributed both to the presence of defects in the structure and to the microstructural refinement $[24,25]$.

Additionally, ECAP may produce textures that improve the H-sorption properties [27, 29,30]. Thus, a recent study examined the influence of preferential orientation in the enhancement of kinetics in the sorption properties of $\mathrm{Mg}$ and $\mathrm{Mg}$ alloys and the (0001) texture was shown to be beneficial for the absorption of hydrogen in bulk materials processed by ECAP [27, 29] and by a combination of ECAP and cold rolling [30]. However, in the two former studies $[27,29]$ the grain size was about one micrometer and in the latter study it was in the range of tens of micrometers [30] so that it was difficult to specifically identify the effect of texture. In all the cases, the samples were left in air to analyze the effect of oxidation and therefore the sample thicknesses were different.

In the AZ31 [27] and ZK60 [29] alloys and commercial purity (CP) Mg [30], the (0001) preferential texture showed its potential by improving the kinetics (by 3.5 times faster on average), absorption and desorption capacities and the resistance to oxidation. For these materials, the times for activation were reduced on average from $\sim 2250 \mathrm{~min}$ to $\sim 660 \mathrm{~min}$ and the shapes of the first absorption curves were typical for a nucleation and growth diffusioncontrolled reaction of the hydride phase. 
The present investigation was initiated specifically to investigate the evolution of the hydrogen sorption kinetics of two magnesium alloys (AM60D and AZ91) with grain sizes in the submicrometer range. Different materials were produced with distinct amounts of a second phase and different misalignments of the (0001) plane with the (0001) pole. For these experiments, all samples had the same thickness and they were polished immediately prior to hydrogenation. These steps were taken to better evaluate the effect of grain size and texture, thereby demonstrating any effect of the synergism between them, together with the influence of any deleterious second phases on the sorption properties.

\section{Experimental materials and procedures}

Experiments were conducted using two commercial coarse-grained magnesium alloys, AM60D and AZ91, supplied by Rima Industrial S/A (Brazil) in the form of ingots. The initial grain sizes in the ingots were similar and $\sim 900 \mu \mathrm{m}$. The chemical compositions of these alloys (in wt.\%) were 5.8\% Al, 0.1\% $\mathrm{Zn}$ and $0.3 \% \mathrm{Mn}$ for $\mathrm{AM} 60 \mathrm{D}$ and $8.4 \% \mathrm{Al}, 0.4 \% \mathrm{Zn}$, and $0.3 \% \mathrm{Mn}$ for AZ91 with the balance as $\mathrm{Mg}$. Thus, it is possible to produce different amounts of second phase in the different samples and thereby analyze their effect on the hydrogenation behavior. The alloys were solution heat treated $\left(24 \mathrm{~h}\right.$ at $\left.425{ }^{\circ} \mathrm{C}\right)$ and then hot extruded to reduce the initial grain size and improve the grain refinement by ECAP. After extrusion, round rods were produced and inspection showed the grain sizes in the as-extruded condition were $\sim 25$ and $\sim 60 \mu \mathrm{m}$ for the AM60D and AZ91 alloy, respectively.

Billets were cut from the rods with lengths of $\sim 60 \mathrm{~mm}$ and these billets were processed by ECAP using a hydraulic press of 150-tons capacity operating at a pressing speed of $\sim 7 \mathrm{~mm} \mathrm{~s}^{-1}$. All processing by ECAP was performed using a solid die having an internal channel angle of $\Phi=90^{\circ}$ and an angle at the outer arc of curvature of the two parts of the channel of $\Psi=20^{\circ}$. These angles produce a strain of $\sim 1.1$ on each separate passage through the die [34] and repetitive pressings were performed using route $\mathrm{Bc}$ in which the 
billet is pressed through the die after rotation by $90^{\circ}$ in the same sense (either clockwise or counterclockwise) between each pass [35].

All billets were pressed at $473 \mathrm{~K}$ but AM60D was pressed for 2 passes to give a total strain of $\sim 2.2$ and AZ91 was pressed for 8 passes to give a total strain of $\sim 8.8$. The temperature during processing was maintained constant to within $+/-2 \mathrm{~K}$. Following ECAP, the processed samples were cut perpendicularly to the pressing direction to reveal the crosssectional plane and then inserted between two AISI 304 stainless steel plates and subjected to 20 passes of cold rolling (CR) using a duo-reversible FENN conventional rolling facility with a $50 \%$ reduction in each pass. This procedure gave a final thickness of $\sim 200 \mu \mathrm{m}$ for all samples.

The hydrogen absorption properties were measured in a Sieverts-type apparatus where the samples were hydrogenated at $573 \mathrm{~K}$ under a hydrogen pressure of $2.0 \mathrm{MPa}$. Prior to hydrogenation, samples were carefully ground and polished to avoid any deformation on the sample surfaces. The desorption properties were evaluated in the same equipment at $573 \mathrm{~K}$ under atmospheric pressure.

An identification of phases was performed by X-ray diffraction (XRD) using monochromatic $\mathrm{K} \alpha$ radiation with an angular pass of $0.032^{\circ}$ in a Rigaku DMAX diffractometer equipped with a C-monochromator. Microstructural characterization and orientation-phase mapping was accomplished by transmission electron microscopy (TEM) using an FEI TECNAI G2 operated at $200 \mathrm{kV}$. This facility was equipped with an orientationphase mapping precession unit NanoMEGAS (model ASTAR) and with a Digistar P1000 unit. To reduce the number of coefficients in the harmonic series expansion, the orthotropic sample symmetry was used in the texture calculations.

\section{Experimental results}

Figures 1 shows typical XRD patterns after processing by ECAP (left) and ECAP + CR (right) for (a) AM60D and (b) AZ91 acquired on the cross-sectional planes of samples 
processed by ECAP and on the longitudinal surfaces of the as-rolled samples. These patterns reveal the presence of $\alpha-\mathrm{Mg}$ and cubic $\mathrm{Mg}_{17} \mathrm{Al}_{12}$. Nevertheless, by comparing the theoretical (inset of Fig.1) and observed relative intensities, it is apparent that the $\alpha-\mathrm{Mg}$ phase has different preferred orientations in these materials for the various processing conditions. In Fig. 1(a) after ECAP, the $\alpha-\mathrm{Mg}$ phase has preferred orientations along the pyramidal $(10 \overline{1} 1)$ plane and the prismatic $(10 \overline{1} 0)$ planes that are activated at high temperatures and become more pronounced on the $(10 \overline{1} 1)$ plane after 2 passes. In Fig. 1(b) for AZ91 the $\alpha-M g$ phase after ECAP has preferred orientations along the pyramidal (10 $\overline{1} 1)$ and basal (0001) planes and is more prominent in the $(10 \overline{1} 1)$ plane after 8 passes. After ECAP + CR, Fig $1(a)$ and (b) shows that the $\alpha-\mathrm{Mg}$ phase has preferred orientations along the basal (0001) plane for both materials and these grow at the expense of all other random orientations and keep constant the theoretical random orientation peak intensity for the prismatic $(10 \overline{1} 0)$ plane. The $(0001)$ orientation is the main slip plane of $\alpha-\mathrm{Mg}$ and, as reported earlier [27-30, 36], is the preferred orientation for hydrogen absorption.

After CR, the relative intensities for the (0001) plane are similar and calculations gave intensity values of $\sim 76 \%$ and $\sim 72 \%$, respectively, for AZ91 and AM60D. However, the combination of all other different residual orientations present in each sample, such as $(10 \overline{1} 2)$ and $(11 \overline{2} 0)$ which are not shown here, suggest that the basal plane is not exactly parallel to the sample normal and in practice it is more tilted in the AM60D alloy.

Figure 1 additionally shows that, as expected, the amount of the second-phase $\left(\mathrm{Mg}_{17} \mathrm{Al}_{12}\right)$ is higher in $\mathrm{AZ91}$. There is a fixed relationship between all of the peaks related to the phase $\mathrm{Mg}_{17} \mathrm{Al}_{12}$ for all conditions in both materials and on average this is close to 1.97 (AZ91/AM60D). In addition, Figure 1 also shows peak broadening after CR which indicates more grain refinement in this alloy.

Figures $2 \mathrm{a}$ and $2 \mathrm{~b}$ show bright field TEM images taken at the cross-sections of samples 
processed by ECAP. After ECAP the grains or subgrains in AM60D (Fig. 2a) are smaller than in AZ91 (Fig. 2b) and this is due to the different initial grain sizes and to the larger number of passes imposed on the AM60D alloy Inspection showed the grain size distribution in the AM60D alloy was multimodal with an average grain/subgrain size of $\sim 0.94 \mu \mathrm{m}$ whereas for the AZ91 alloy there was a regular distribution with an average grain size of $\sim 1.85 \mu \mathrm{m}$. It is apparent from Figs. 2a and 2b that AM60D has the largest number of dislocations within the grains whereas the amounts of second phase particles, both within the grains and in the grain boundaries, were higher in theAZ91 alloy.

Figures $2 \mathrm{c}$ and $2 \mathrm{~d}$ show bright field TEM images taken at the longitudinal sections of samples processed by ECAP + CR. After processing, the grains or subgrains in AM60D (Fig. 2c) are smaller than in AZ91 (Fig. 2d). Measurements gave average grain/subgrain sizes of $\sim 310 \mathrm{~nm}$ in AM60D and $\sim 550 \mathrm{~nm}$ in AZ91 so that there was an average reduction of only $\sim 3$ times after $20 \mathrm{CR}$ passes. This shows that CR is less efficient than ECAP for achieving grain refinement. In addition, in Figs. $2 \mathrm{c}$ and $2 \mathrm{~d}$ there are elongated grains in AM60D and boundaries are present within large grains in AZ91. This makes it difficult to determine the grain size and therefore orientation mapping was conducted using TEM in the longitudinal sections of the alloys processed by ECAP $+\mathrm{CR}$. This type of mapping is analogous to using electron backscatter diffraction (EBSD) in a scanning electron microscopy (SEM) except that it has a higher resolution and may be used conveniently with nanometric grain sizes.

Figure 3 shows virtual bright field (BF) images, equivalent to those obtained by conventional TEM, superimposed with misorientation information in the form of red lines for low-angle grain boundaries (LAGBs) and blue lines for high-angle grain boundaries (HAGBs). Figures $3 \mathrm{a}$ and $3 \mathrm{~b}$ are for longitudinal sections of AM60D and AZ91 after processing by ECAP+CR and Figs $3 \mathrm{c}$ and $3 \mathrm{~d}$ show second phases in the longitudinal sections for AM60D and AZ91, respectively. These images confirm a non-homogeneous distribution 
of grains/subgrains in both alloys but the appearance of AM60D (Fig. 3a) suggests a multimodal distribution of grains when compared to AZ91 (Fig. 3b). The misorientation angles of the grain boundaries were indexed and Fig. 3 shows HAGBs (blue) with misorientation angles equal to or greater than $15^{\circ}$ and LAGBs (red) having misorientation angles less than $15^{\circ}$. By indexing these boundaries for the $\alpha-\mathrm{Mg}$ phase, it can be seen that both samples contain almost the same fraction of HAGBs ( 83\% and $\sim 77 \%$ for AM60D and AZ91, respectively).

The size distributions of (a) grains and (b) second phase are shown in Fig. 4 for samples processed by ECAP + CR for the longitudinal sections of the AM60D and AZ91 alloys. The measured average grain sizes were 261 and $492 \mathrm{~nm}$ for AM60D and AZ91, respectively, and this is smaller than the values obtained from the images in Fig. 2. The average second phase particle sizes were $\sim 91$ and $\sim 123 \mathrm{~nm}$ for AM60D and AZ91, respectively. However, due to the compositional difference, there is a significant difference in the amounts of second phase between AM60D and AZ91. Measurements obtained from either non-red regions in Figs. 3c and $3 \mathrm{~d}$ or black regions in Fig. 5 gave area fractions of $\sim 11 \%$ and $\sim 23 \%$ for AM60D and AZ91, respectively, where Fig. 5 shows orientation imaging mappings (OIM) for samples after processing by ECAP + CR for (a) AM60D and (b) AZ91. In Fig. 5 the second phase was removed and the black regions represent positions of the second phase. It is apparent that there is a greater tendency for red in Fig. 5b than 5a which means there is a stronger tendency for an (0001) preferential texture in AZ91 than in AM60D.

Figure 6 shows pole figures for the samples after processing by ECAP + CR for longitudinal sections of (a) AM60D and (b) AZ91. These pole figures reveal a tendency for a texture in the strong preferential direction of (0001) for H-sorption. However, a more intense texture is present in AZ91 where the intensity is $~ 18$ times the random value whereas it is only 13 times the random value for AM60D. This is the same trend observed in the X-ray 
diffraction spectra in Fig. 1 and in the OIMs in Fig. 5. However, it is noteworthy that the acquired texture in AZ91 is near to the (0001) pole around $23^{\circ}$ from the pole which means that the (0001) poles are off the sample normal. By contrast, the (0001) poles in AM60D are about $63^{\circ}$ from the sample normal. The other orientations are randomly distributed along their poles in both samples.

The absorption kinetic measurements are presented in Fig. 7a where the hydrogen absorption is plotted against time. These plots compare the first absorption curves between the longitudinal sections for AM60D and AZ91 after processing by ECAP + CR. Examination shows that a much faster hydrogenation kinetic (inset of Fig. 7a) is observed for the sample with a considerably stronger preferential texture as in the longitudinal section in AZ91 where the (0001) pole is off the sample normal. The maximum hydrogen contents were $\sim 4.7$ wt.\% after only $\sim 15 \mathrm{~h}$ in the longitudinal section of AZ91 and $\sim 6.3 \mathrm{wt} . \%$ after $\sim 40 \mathrm{~h}$ in the longitudinal section of AM60D. To emphasize the difference, it should be noted that the amount of hydrogen in AM60D was only $~ 1.6 \mathrm{wt} . \%$ after $15 \mathrm{~h}$, the equivalent to $25 \%$ of the maximum capacity. These different kinetics for hydrogen absorption are mostly due to the significant differences in the texture behavior of the two alloys.

Fig. $7 \mathrm{~b}$ shows the desorption properties of the hydrogenated samples processed by ECAP + CR. From these plots, it is evident that the amount of desorbed hydrogen is almost the same as the absorbed amounts shown in Fig. 7a. However, the kinetic of desorption for AM60D is faster than for AZ91. Inspection of the inset in Fig. 7b suggests this effect may be due to the differences between the desorbed amounts of each sample because the maximum transformed fraction is reached more quickly in AZ91 than in AM60D.

\section{Discussion}

The results presented in Fig. 7a and in its inset clearly demonstrate a very large difference in the absorption kinetics of the samples. These different kinetics for hydrogen 
absorption are ascribed to the significant differences in the texture behavior of the two samples and consequently to the preferred texture that is available in specific directions.

It is interesting to note that, even having a smaller grain size (Fig. 4a), AM60D has the less satisfactory texture and possesses slower kinetics than AZ91, thereby demonstrating again the advantage of a good combination between the nanosized grains and the texture. This was not entirely the situation for AZ91 where the average grain size was almost double the value for AM60D. However, texture compensated for this difference leading to very fast first absorption in AZ91. It may be argued that AZ91 was more deformed than AM60D and yet both samples had similar LAGBs (Fig. 3) which means that the final deformed state was similar for both samples.

It is also important to note that, for both samples, although there is the presence of a preferred texture, the ability for hydrogen absorption is substantially lower than the theoretical calculated capacities for both alloys of $7.1 \mathrm{wt} . \%$ and $6.9 \mathrm{wt} \%$ for AM60D and AZ91, respectively. For reference, AM60D absorbed $~ 87 \%$ of the maximum capacity and AZ91 only $\sim 66 \%$. This behavior cannot be related to thickness differences $[27,30]$ because all samples had the same thickness. This behavior may be due to the difference in the amounts of second phases in each specimen ( 11\% and $23 \%$ for AM60D and AZ91, respectively) since this decreases the surface for the hydrogen penetration in both samples but more in AZ91 than in AM60D.

The inset of Figure 7a represents the transformed fractions for both alloys which were constructed by normalizing the maximum acquired capacity as $100 \%$ of the transformation. The shapes of the transformation curves present a sigmoidal shape and, from the original theory of transformation [37], this type of curve is usually related to the nucleation and growth reactions where a region is formed during a time equal to $\tau$ (an induction period) after a reaction initiation but before a monotonically growing curve. It is noteworthy that 
sigmoidal curves are also considered as a result of an oxide layer on the sample surface [36], but in these experiments the samples were polished before hydrogenation.

The reaction of formation of a metal hydride is generally complex. Usually, it is divided into at least four simplified reactions [4, 5, 38-40]: (i) Dissociation/adsorption; (ii) Surface penetration; (iii) Bulk diffusion; (iv) Hydride formation. The nucleation and growth kinetics are generally described by the Johnson-Mehl-Avrami (JMA) equation [41-44]:

$$
x(t)=1-\exp \left(-(k t)^{n}\right)
$$

where $x(t)$ is the transformed fraction, $k$ is the kinetic rate constant (nucleation and growth rates) and $t$ is the reaction time. The Avrami exponent (n) then describes the type of nucleation, the dimensionality of growth, and its rate-limiting step. The slope of the straight line formed when plotting $\ln [\ln (1 /(1-x(t)))] v s . \ln (t)$ gives the value of $\mathrm{n}$.

Figure 8 presents the resulting curves after applying the JMA model to the curves of the inset of Figure $7 \mathrm{a}$ where these curves show three linear regions for both alloys. These regions were individually fitted to a suitable JMA function [41-44] to provide the best fit to the whole set of experimental data. It is worth mentioning that two linear regions were covered earlier [37] and this was observed in earlier work [30] and related to the presence of a strong preferential textures. For all conditions, site saturation can be assigned to the last linear region [37] and such saturation phenomenon will strongly influence the growth of hydride nuclei and thereby lead to a substantial reduction in the transformation rate.

The values obtained for the Avrami exponent (n) and the rate constant (k) for each JMA function are given in Fig. 8 (n1 and $\mathrm{k} 1$ refer to the first linear region and $\mathrm{n} 2$ and $\mathrm{k} 2$ to the second region). From Fig, 8 it is apparent that, when the hydrogenation starts, there is a large difference between n1 values for the two alloys. For AZ91 which possesses stronger texture and is more aligned to the (0001) pole than AM60D (Fig. 6), the value of $n 1$ is $~ 0.5$ whereas for AM60D it is $\sim 1.5$. 
It is interesting to note the influence of grain size. For any alloy, and any condition of texture, the observed values for $\mathrm{n} 1$ denote that diffusion is the rate-limiting step. This means that grains grow one-dimensionally with decreasing nucleation rate. However, for AZ91, with larger grain sizes and smaller surface area, the value of $\sim 0.5$ indicates a thickening of large plates [37] after complete edge impingement of grains and this suggests difficulties for hydrogen penetration during the short induction period $(\tau)$ of hydrogenation. This means that nucleation is mostly on the sample surface and indeed this very small value of $n 1$ indicates that nucleation and growth during $\tau$ is slow.

By contrast, for AM60D where the grain size is much smaller, the one-dimensionality for $n 1=1.5$ is evidence of growing in grain boundaries, as this value suggests that all shapes are growing from small dimensions but with zero nucleation rate [37]. Initially the reaction is faster and probably the hydrogen dissociation is easier because of the higher volume of interfaces. However, the rate constant k1 for AM60D indicates that the nucleation and growth rates ae very slow and the nucleation rate is zero. Thus, even with a higher value of $n 1$ this indicates a faster reaction and denotes difficulties for hydrogen penetration which justifies the long induction period. On the other hand, k1 for AZ91 was almost one order of magnitude larger than for AM60D indicating that the nucleation and growth rates were almost ten times larger in AZ91 because the nucleation and growth were mostly on the sample surface.

The shorter induction period for AZ91 indicates that the hydrogen was able to penetrate faster in this sample than in AM60D. This behavior is justified by the texture (Fig. 6) in sample AZ91 around $23^{\circ}$ from the (0001) pole compared with $63^{\circ}$ for AM60D together with the observed stronger texture in AZ91 (18 times for AZ91 against 13 times for AM60D). This means that it was easier for the hydrogen to find positions of less energy for dissociation in AZ91 than in AM60D. 
After the induction period (first linear region), there is a change in slopes in the JMA curves going to the second linear region. This change is fast and smooth for AZ91 taking about 30 minutes whereas it is slow and drastic for AM60D taking about 13 hours to start changing. Interestingly, the second linear regions for both samples are similar and they both possess almost the same values for $\mathrm{n} 2$ and $\mathrm{k} 2$. This similarity is an indication that AM60D finally started to be hydrogenated following the preferential texture which reflects the lower energy of texture for hydrogen dissociation than boundaries and defects. The value of n2 increased from $\sim 0.5$ to $\sim 0.9$ for AZ91 and decreased from $\sim 1.5$ to $\sim 0.9$ for AM60D while k2 increased for both alloys reaching almost the same value of $\sim 0.13$. However, $\mathrm{k} 2$ increased by almost two order of magnitude for AM60D against one order of magnitude for AZ91.

Although there are differences in the $\mathrm{n} 2$ values, they indicate that the rate-limiting step is diffusion. However, these values may mean the growing of needles and plates of finite long dimensions (but small by comparison with their separation) or the thickening of long cylinders or needles [37] as in hydride growing through the surface of the magnesium grains following the preferential texture. Furthermore, the reactions were much faster, making the hydrogen dissociation easier and thereby allowing hydrogen penetration in an easier way. Despite this, the nucleation and growth rates for AM60D were $\sim 36 \%$ smaller than for AZ91, thereby justifying the longer time for AM60D to reach the maximum capacity

The results presented in Fig. 7b, which show desorption properties for the hydrogenated samples processed by ECAP $+\mathrm{CR}$, demonstrate that the amount of desorbed hydrogen was almost the same as the absorbed amounts shown in Fig. 7a. At first a glance, it appears that the kinetic of desorption for AM60D was faster than for AZ91. However, this analysis may be influenced by differences between the desorbed amounts. Inspection of the inset of Fig. $7 \mathrm{~b}$, which presents the transformed desorption fraction, demonstrates that both have similar desorption kinetics. Nevertheless, probably due to the effect of grain size, the 
AM60D desorbed slightly faster than AZ91 until about one hour. Thereafter, the preferred texture of AZ91 accelerated the reaction at the final stage of desorption.

These results demonstrate that, after processing by ECAP $+\mathrm{CR}$, different hydrogen sorption results may be attained primarily due to differences in texture acting synergistically with the grain size distributions. Second phases, which do not contribute as catalysts, may be deleterious for the acquired capacity. This is confirmed by the different amounts of second phases in the two different magnesium alloys.

\section{Summary and conclusions}

1. Commercial magnesium AM60D and AZ91 extruded alloys, with grain sizes of $\sim 25$ and $\sim 60 \mu \mathrm{m}$, respectively, and having different amounts of second phase, were processed by ECAP under different conditions of numbers of passes. After ECAP, the grain sizes were measured as $\sim 0.94$ and $\sim 1.85 \mu \mathrm{m}$ for AM60D and AZ91, respectively. Following ECAP, CR was performed for 20 passes giving grain sizes of $\sim 261$ and $~ 492 \mathrm{~nm}$, respectively. However, these alloys acquired different amounts of (0001) preferential orientation and this was larger and more aligned with the (0001) pole in AZ91.

2. The capacity of CR to refine the grains is marginal compared with ECAP but the simplicity of processing makes it more effective in producing a preferential texture. The samples showed different hydrogen capacities of $\sim 4.6 \mathrm{wt} \%$ and $\sim 6.2 \mathrm{wt} \%$ for AZ91 and AM60D, respectively. There was an influence of texture on the kinetics so that for AZ91 the capacity was reached in $\sim 15 \mathrm{~h}$ whereas for AM60D it was reached in $\sim 40 \mathrm{~h}$. These capacities are low and correlate with the thickness of the samples. The difference in the capacities is attributed to the presence of dissimilar amounts of second phases.

3. During absorption, AM60D with a smaller grain size and poorer texture gave a greater Avrami exponent during the first stage of hydrogenation than AZ91 with a larger grain size and better texture. This means there is an accelerated reaction, thereby 
demonstrating the importance of the ultrafine grains in the absorption. During desorption, in the course of the early stages, the grain size influenced the kinetics more than the texture. In the later stages, the texture dominated the process.

4. It is concluded that a good combination of ultrafine grains and texture may produce very promising sorption properties. Thus, it is proposed that promising new applications related to hydrogen storage may be investigated by using ECAP + CR to fabricate $\mathrm{Mg}$ and Mg-based alloys with exceptionally small grain sizes and by evaluating the optimum procedure for achieving a preferential texture.

\section{Acknowledgements}

This work was conducted in cooperation with the University of Southampton (U.K.), Ufa State Aviation Technical University (Russia) and LEPMI Laboratory, Université Joseph Fourier, Grenoble (France). It was supported in part by award FAPESP\# 2010/11477-4, in part by award FAPESP\# 2011/51245-8 under a cooperation agreement between the Federal University of Sao Carlos and the University of Southampton, in part by award FAPESP TEMATIC\# 2013/05987-8 and in part by the Conselho Nacional de Desenvolvimento Cientifico e Tecnológico CNPq\# 237345/2012-9 PDE (CsF). One of the authors gratefully acknowledges Fundação de Amparo à Pesquisa do Estado de São Paulo (Brazil) under grant number FAPESP\# 2012/13179-6 (AMJ). 


\section{References}

[1] Sakintuna B, Lamari-Darkrim F, Hirscher M. Metal hydride materials for solid hydrogen storage: a review. Int J Hydrog Energy 2007;32:1121-40.

[2] Schlapbach L, Zuttel A. Hydrogen-storage materials for mobile applications. Nature 2001;414:353e8.

[3] Z. Wu, F. Yang, Z. Bao, S.N. Nyamsi, Z. Zhang. Improvement in hydrogen storage characteristics of Mg-based metal hydrides by doping nonmetals with high electronegativity: a first-principle study. Comput Mater Sci. 2013;78:83-90

[4] Topler J, Buchner H, Saufferer H, Knorr K, Prandl W. Measurements of the diffusion of hydrogen atoms in magnesium and $\mathrm{Mg} 2 \mathrm{Ni}$ by neutron scattering. $\mathrm{J}$ Less-Common Met $1982 ; 88: 397-404$

[5] Huot J, Liang G, Boily S, Van Neste A, Schulz R. Structural study and hydrogen sorption kinetics of ball-milled magnesium hydride. J Alloys Compds 1999;293:495-500.

[6] Manchester FD, Khatamian D. Mechanisms for activation of intermetallic hydrogen absorbers. Mater Sci Forum 1988;31:261-9.

[7] Zaluska A, Zaluski L, Stro“meOlsen JO. Nanocrystalline magnesium for hydrogen storage. J Alloys Compds 1999;288:217-25.

[8] P. Hjort, A. Krozer, B. Kasemo, J. Alloys Comp. 1996;237:74-.

[9] B.Vigeholm, J. Kjo“ller, B. Larsen, A.S. Pedersen, J. Less-Common Met.1984;104:141-. [10] A. Krozer, B. Kasemo, J. Vac. Sci. Technol. 1987;A5:1003-.

[11] B.Vigeholm, J. Kjo“ller, B. Larsen, J. Less-Common Met. 74 (1980) 341

[12] L. Belkbir, E. Joly, N. Gerard, Int. J. Hydrogen Energy 6 (1981) 285

[13] Fujii H, Higuchi K, Yamamoto K, Kajioka H, Orimo S, Toiyama K. Remarkable hydrogen storage, structural and optical properties in multi-layered $\mathrm{Pd} / \mathrm{Mg}$ thin films. Mater Trans 2002;43(11):2721e7. 
[14] Gennari FC, Castro FJ, Urretavizcaya G. Hydrogen desorption behavior from magnesium hydrides synthesized by reactive mechanical alloying. J Alloys Compds 2001;321:46e53.

[15] Higuchi K, Yamamoto K, Kajioka H, Toiyama K, Honda M, Orimo S, et al. Remarkable hydrogen storage properties in three-layered $\mathrm{Pd} / \mathrm{Mg} / \mathrm{Pd}$ thin films. $\mathrm{J}$ Alloys Compds 2002;330-332:526-30.

[16] B. Tanguy, J.L. Soubeyroux, M. Pezat, J. Portier, P. Hagenmu“ller, Mat. Res. Bull. 11 (1976) 1441.

[17] S. Bouaricha, J.P. Dodelet, D. Guay, J. Huot, S. Boily, R. Schulz, J. Alloys Comp. 297 (2000) 282.

[18] G. Liang, J. Huot, S. Boily, A. Van Neste, R. Schulz, J. Alloys Comp. 291 (1999) 295.

[19] Oelerich W, Klassen T, Bormann R. Metal oxides as catalysts for improved hydrogen sorption in nanocrystalline Mg-based materials. J Alloy Compds 2001;315:237e42.

[20] Oelerich W, Klassen T, Bormann R. Comparison of the catalytic effects of V, V2O5, $\mathrm{VN}$, and $\mathrm{VC}$ on the hydrogen sorption of nanocrystalline Mg. J Alloys Compds 2001;322:L5e9.

[21] Rivoirard S, de Rango P, Fruchart D, Charbonnier J, Vempairea D. Catalytic effect of additives on the hydrogen absorption properties of nano-crystalline $\mathrm{MgH} 2(\mathrm{X})$ composites. $\mathrm{J}$ Alloys Compds 2003;356e357:622e5.

[22] Imamura H, Masanari K, Kusuhara M, Katsumoto H, Sumi T, Sakata Y. High hydrogen storage capacity of nanosized magnesium synthesized by high energy ball-milling. $\mathbf{J}$ Alloys Compds 2005;386:211e6

[23] N.A.A. Rusman, M. Dahari. International J. Hyd. Ener.41 (2016) 12108

[24] Skripnyuk VM, Rabkin E, Estrin Y, Lapovok R. Improving hydrogen storage properties of magnesium based alloys by equal channel angular pressing. Int $\mathbf{J}$ Hydrog Energy 
2009;34:6320e4.

[25] Krystian M, Zehetbauer MJ, Kropik H, Mingler B, Krexner G. Hydrogen storage properties of bulk nanostructured ZK60 Mg alloy processed by equal channel angular pressing. J Alloys Compds 2011;509:S449e55.

[26] Edalati K, Yamamoto A, Horita Z, Ishihara T. High-pressure torsion of pure magnesium: evolution of mechanical properties, microstructures and hydrogen storage capacity with equivalent strain. Scr Mater 2011;64:880e3.

[27] Jorge Jr AM, Prokofiev E, de Lima GF, Rauch E, Veron M, Botta WJ, et al. An investigation of hydrogen storage in a magnesium-based alloy processed by equal-channel angular pressing. Int J Hydrog Energy 2013;38:8306e12.

[28] Botta WJ, Jorge Jr AM, Veron M, Rauch E.F., Ferrie E, Yavari AR, et al. H-sorption properties and structural evolution of $\mathrm{Mg}$ processed by severe plastic deformation. $\mathrm{J}$ Alloy Compds 2013;580:S187-91.

[29] Prokofiev, E.A.; Jorge, A.M. ; Botta, W.J. ; Valiev, R.Z. . Development of UltrafineGrained Metals by Equal-Channel Angular Pressing. In: Chester J. Van Tyne; Gilmar F. Batalha; Sergio Button; David Cameron; Joseph McGeough; Emin Bayraktar; Bekir Yilbas; Nabil Bassim; Bekir Yibas; Syed Masood; Mustafizur Rahman; George Krauss; Saleem Hashmi. (Org.). Comprehensive Materials Processing. 1ed.: Elsevier, 2014, v. 3, p. 187-209. [30] Alberto Moreira Jorge Jr., Gisele Ferreira de Lima, Maria Regina Martins Triques, Walter Jose’ Botta, Claudio Shyinti Kiminami, Ricardo Pereira Nogueira, Alain Reza Yavari, Terence G. Langdon. Correlation between hydrogen storage properties and textures induced in magnesium through ECAP and cold rolling. . Int J Hydrog Energy 2014;39:3810-21.

[31] Valiev RZ, Islamgaliev RK, Alexandrov IV. Bulk nanostructured materials from severe plastic deformation. Prog Mater Sci 2000;45:103e89. 
[32] Valiev RZ, Langdon TG. Principles of equal-channel angular pressing as a processing tool for grain refinement. Prog Mater Sci 2006;51:881e981.

[33] Zhilyaev AP, Langdon TG. Using high-pressure torsion for metal processing: fundamentals and applications. Prog Mater Sci 2008;53:893e979.

[34] Y. Iwahashi, J. Wang, Z. Horita, M. Nemoto, T.G. Langdon. Principle of equal-channel angular pressing for the processing of ultra-fine grained materials. Scripta Mater. 1996;35:143-6.

[35] M. Furukawa, Y. Iwahashi, Z. Horita, M. Nemoto, T.G. Langdon. The shearing characteristics associated with equal-channel angular pressing. Mater. Sci. Eng. A $1998 ; 257: 328-32$.

[36] A. Léon, E.J. Knystautas, J. Huot, R. Schulz. Influence of the evaporation rate and the evaporation mode on the hydrogen sorption kinetics of air-exposed magnesium films. Thin Solid Films 2006;496:683-7

[37] Christian JW. The theory of transformations in metals and alloys. 2nd ed. Oxford: Pergamon; 1975.

[38] Wang CS, Wang XH, Lei YQ, Chen CP, Wang QD. The hydriding kinetics of MINi5-I. Development of the model. Int. J. Hydrogen Energy 1996;21:471-8.

[39] Chou K-C, Li Q, Lin Q, Jiang L-J, Xu KD. Kinetics of absorption and desorption of hydrogen in alloy powder. Int. J. Hydrogen Energy 2005;30:301-9.

[40] Danaie M, Mauer C, Mitlin D, Huot J. Hydrogen storage in bulk Mg-Ti and Mgstainless steel multilayer composites synthesized via accumulative roll-bonding (ARB). Int. J. Hydrogen Energy 2011;36:3022-36

[41] Johnson WA, Mehl RF. Reaction kinetics in processes of nucleation and growth. Trans AIME 1939;135:416-58.

[42] Avrami M. Kinetics of phase change. I, general theory. J Chem Phys 1939;7:1103-12 
[43] Avrami M. J. Kinetics of phase change. II, transformation time relations for random distribution of nuclei. Chem Phys 1940;8:212-24.

[44] Avrami M. Granulation, phase change, and microstructure, kinetics of phase change. III. J Chem Phys 1941;9:177-84. 
Figure Captions

Figure 1 - XRD patterns of Mg alloys comparing samples processed by ECAP (left) and ECAP+CR (right) for: (a) the cross-section of sample AM60D and (b) the longitudinalsection of sample AZ91. The inset (center of the Figure) shows the theoretical relative intensities for non-textured $\alpha-\mathrm{Mg}$.

Figure 2 - Bright field TEM images taken at: a cross-section of the samples processed by ECAP, AM60D in (a) and AZ91 in (b), respectively; and at a longitudinal section of the samples processed by ECAP+CR, AM60D in (c) and AZ91 in (d), correspondingly.

Figure 3 - Virtual bright field images obtained by TEM using the NanoMEGAS Astar system for (a) the AM60D sample (longitudinal section). (b) the AZ91 sample (longitudinal-section). (c) second phase in AM60D. (d) second phase in AZ91. Red represents the magnesium matrix in ( $a$ and b). High-angle boundaries are represented by blue lines (misorientation angles $>15^{\circ}$ ) and low-angle grain boundaries are represented by red lines (misorientation angles $<15^{\circ}$ ).

Figure 4 - Size distributions of grains (a) and second-phase (b) after processing by ECAP+CR for the longitudinal sections of the AM60D and AZ91 alloys.

Figure 5 - Orientation imaging mappings (OIM) for the samples after processing by ECAP+CR of (a) for the longitudinal section of the AM60D alloy and (b) for the longitudinal-section of the AZ91 alloy.

Figure 6 - Pole figures for the processed samples after ECAP+CR of (a) for the longitudinal 
section of the AM60D alloy and (b) for the longitudinal-section of the AZ91 alloy.

Figure 7 - (a) Hydrogen absorption at $573 \mathrm{~K}$ under $2 \mathrm{MPa}$ of $\mathrm{H}_{2}$ after processing by ECAP+CR for the AZ91 and AM60D alloys, the inset represents the absorption transformed fraction. (b) Hydrogen desorption at $573 \mathrm{~K}$ under atmospheric pressure, the inset represent the desorption transformed fraction.

Figure 8 - Plotting of $\ln [\ln (1 /(1 \mathrm{~L} x(\mathrm{t})))]$ vs. $\ln (\mathrm{t})$. JMA model applied to curves of the inset of Figure 7a, for the condition of ECAP+CR for the AZ91 and AM60D alloys 

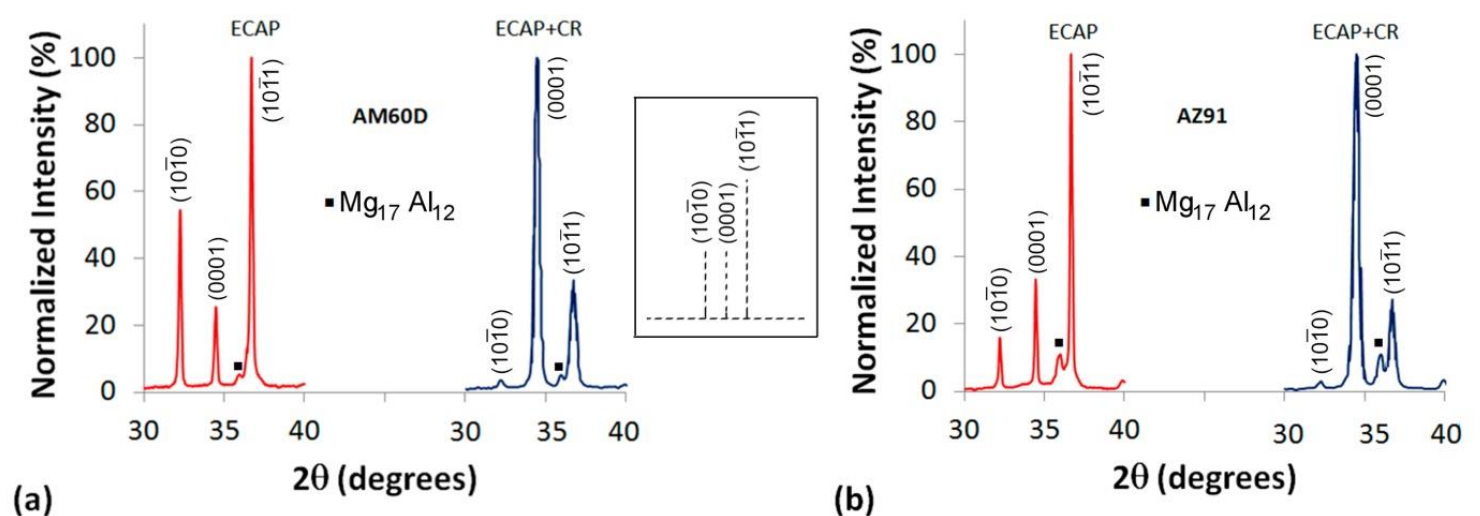

Figure 1

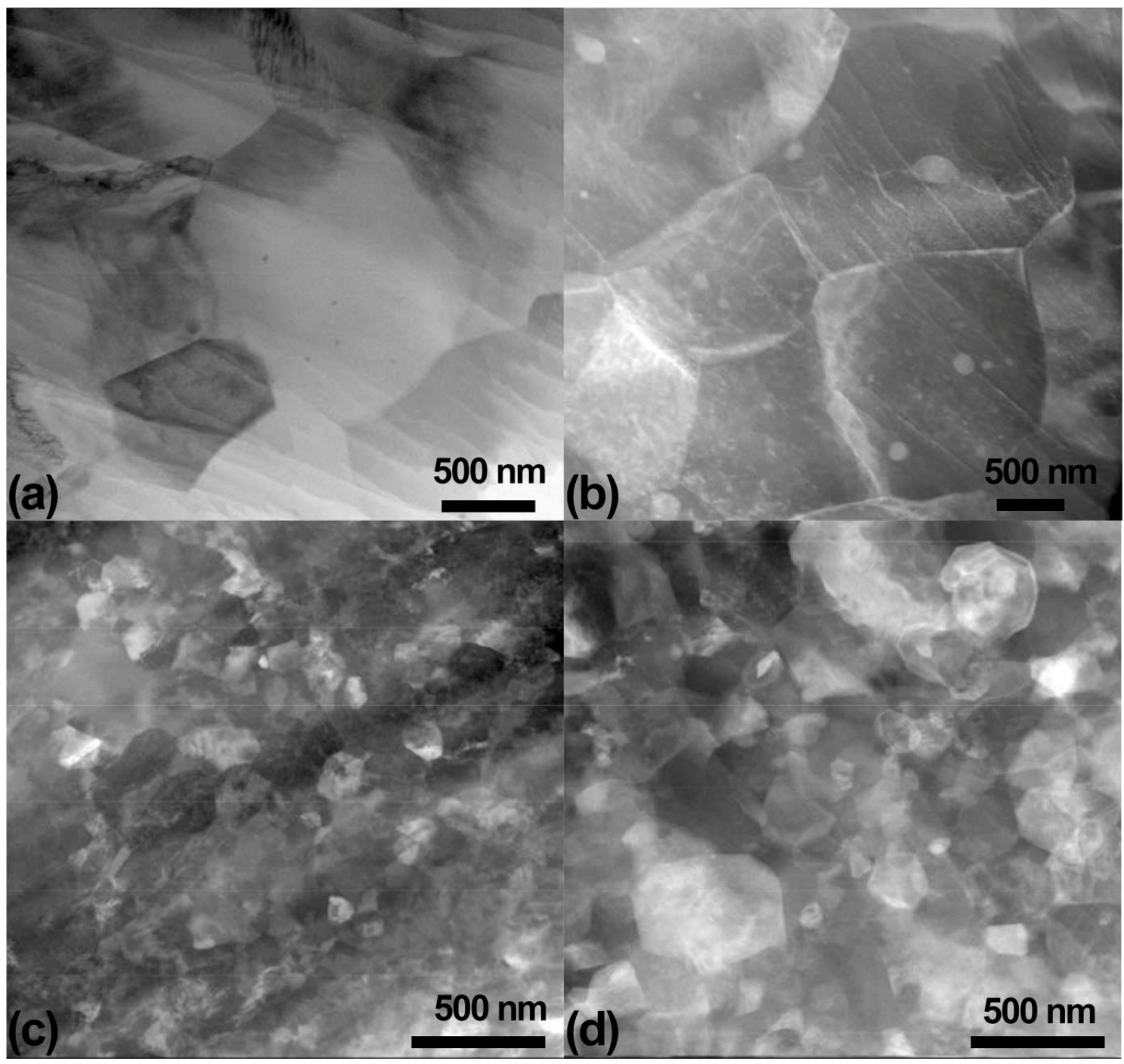

Figure 2 


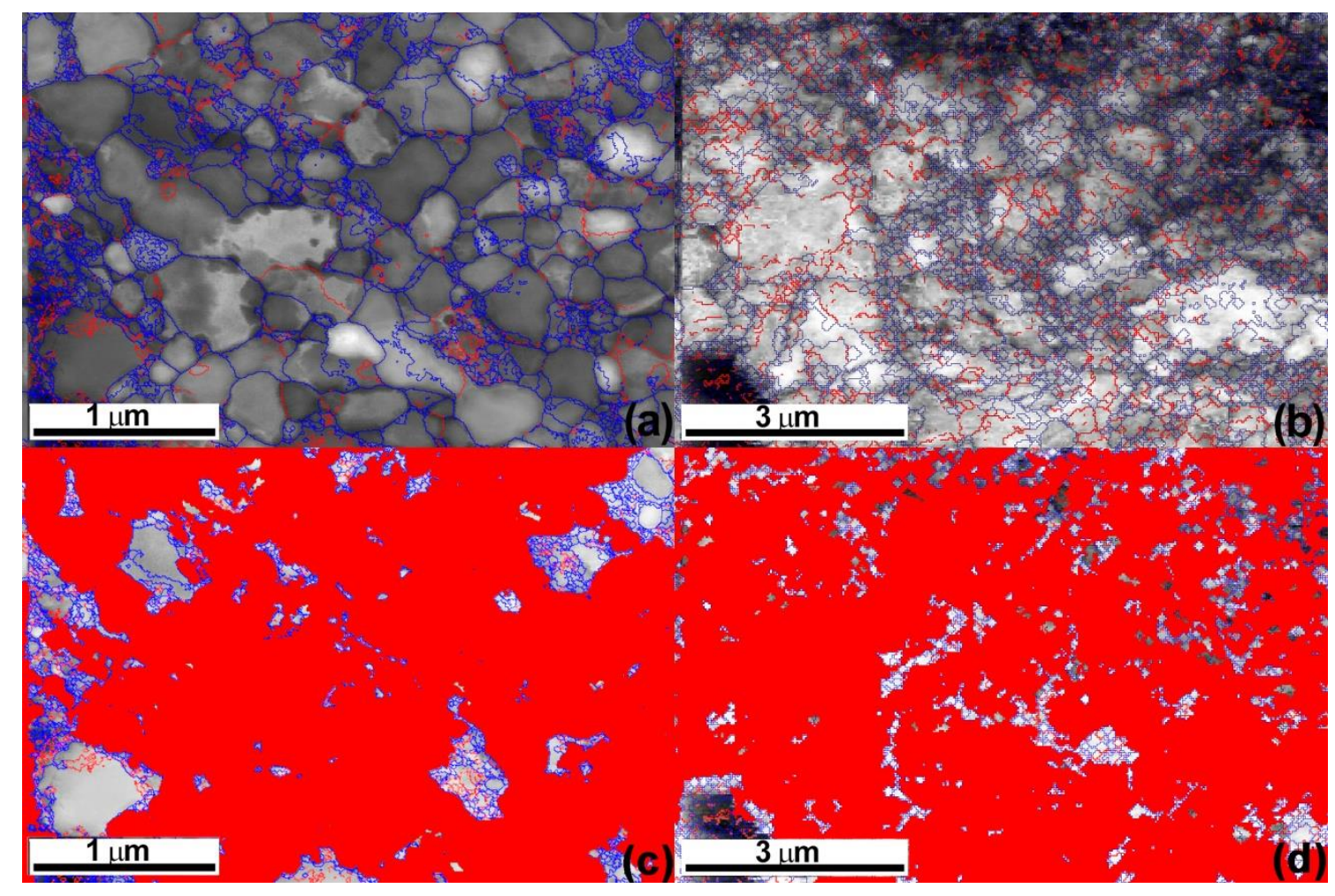

Figure 3
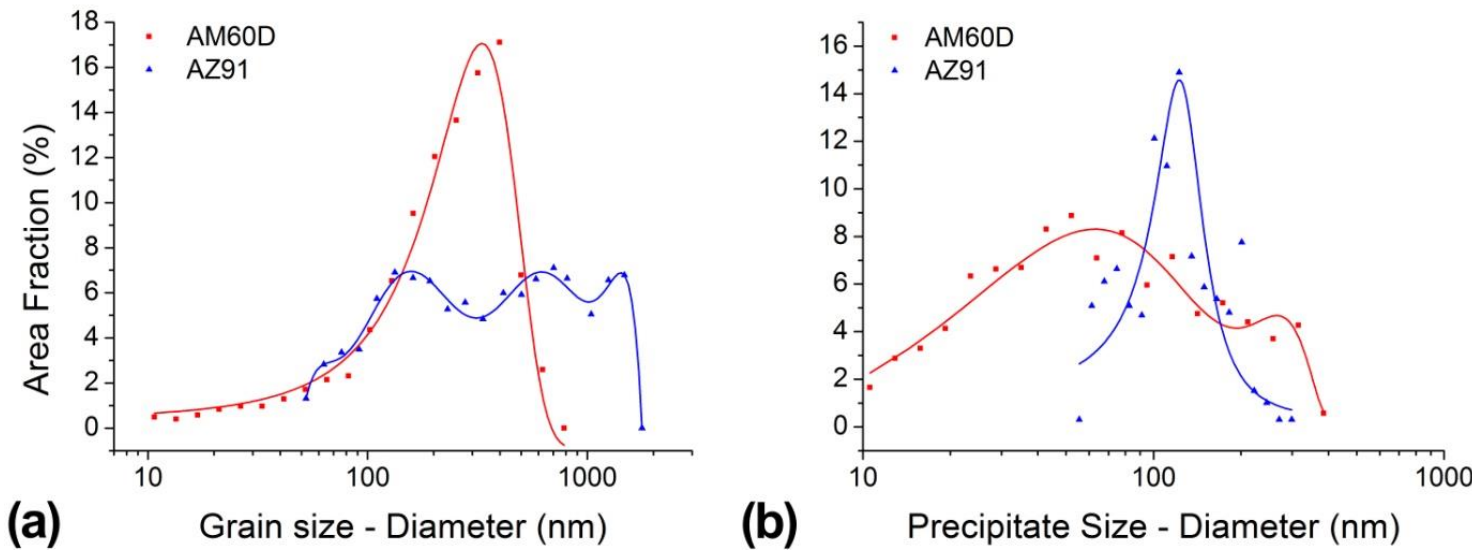

Figure 4 


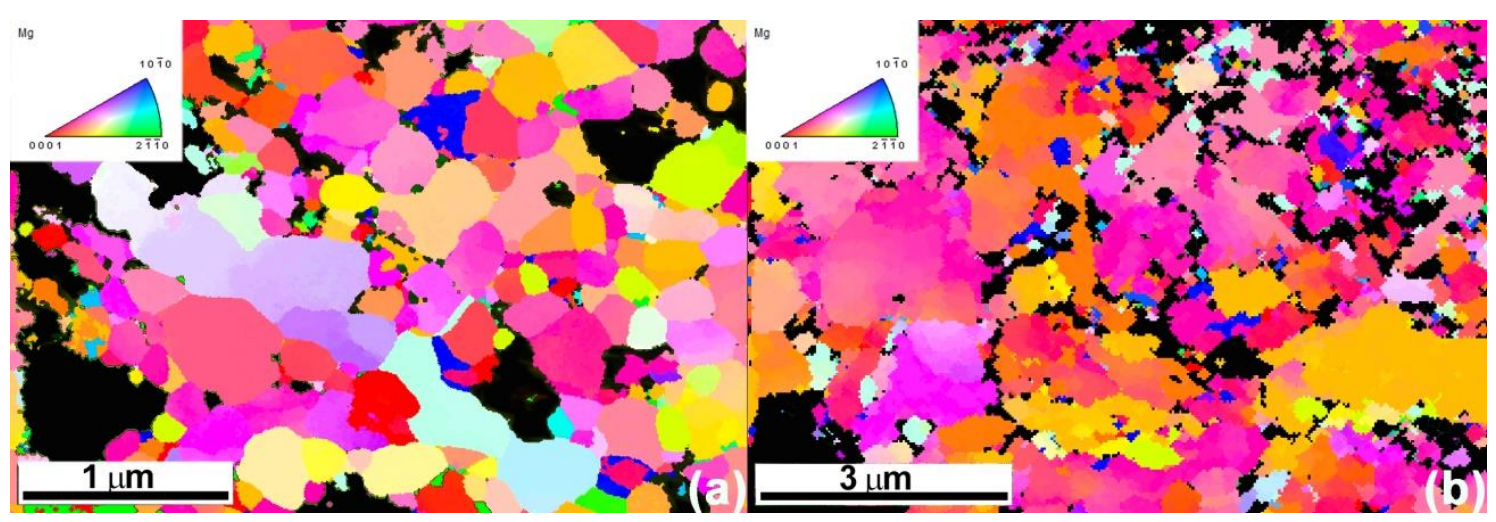

Figure 5

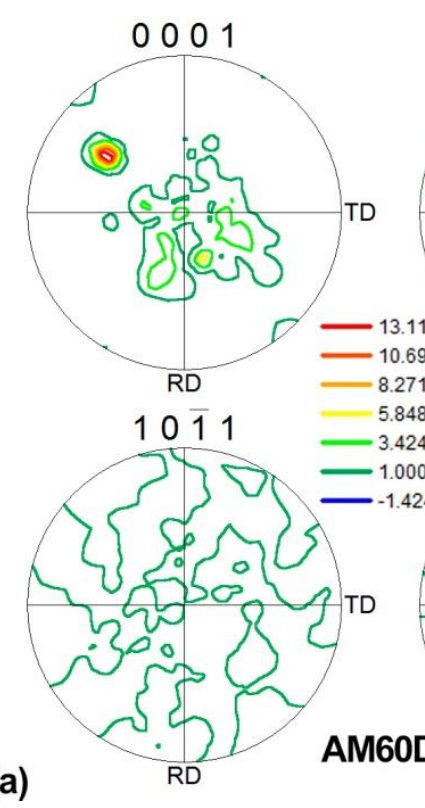

(a)
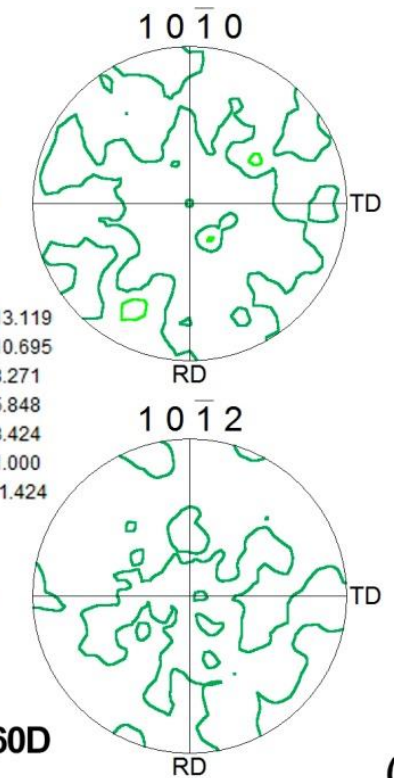

(b)
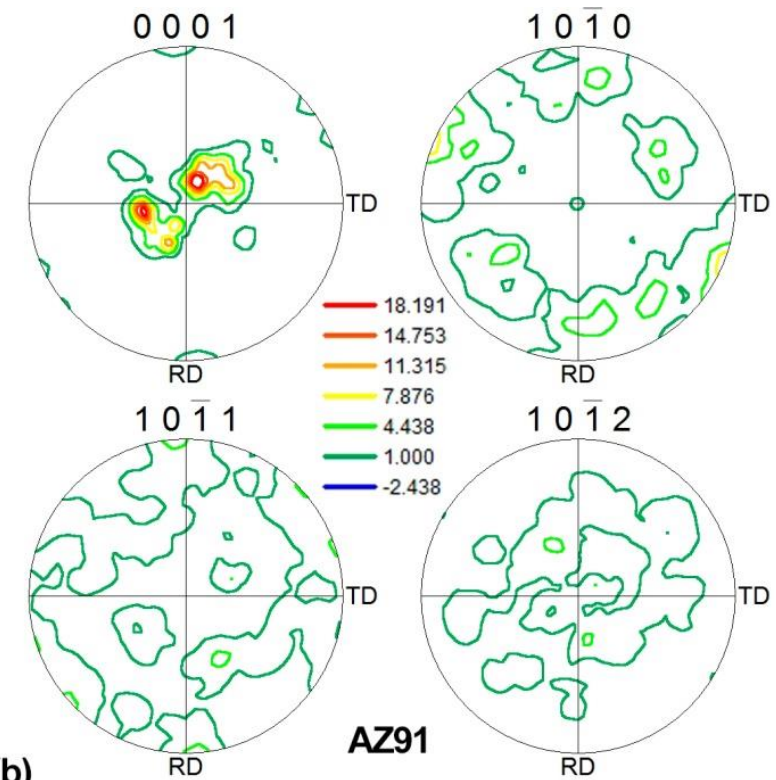

AZ91

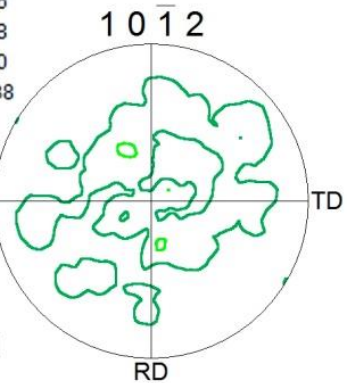

Figure 6
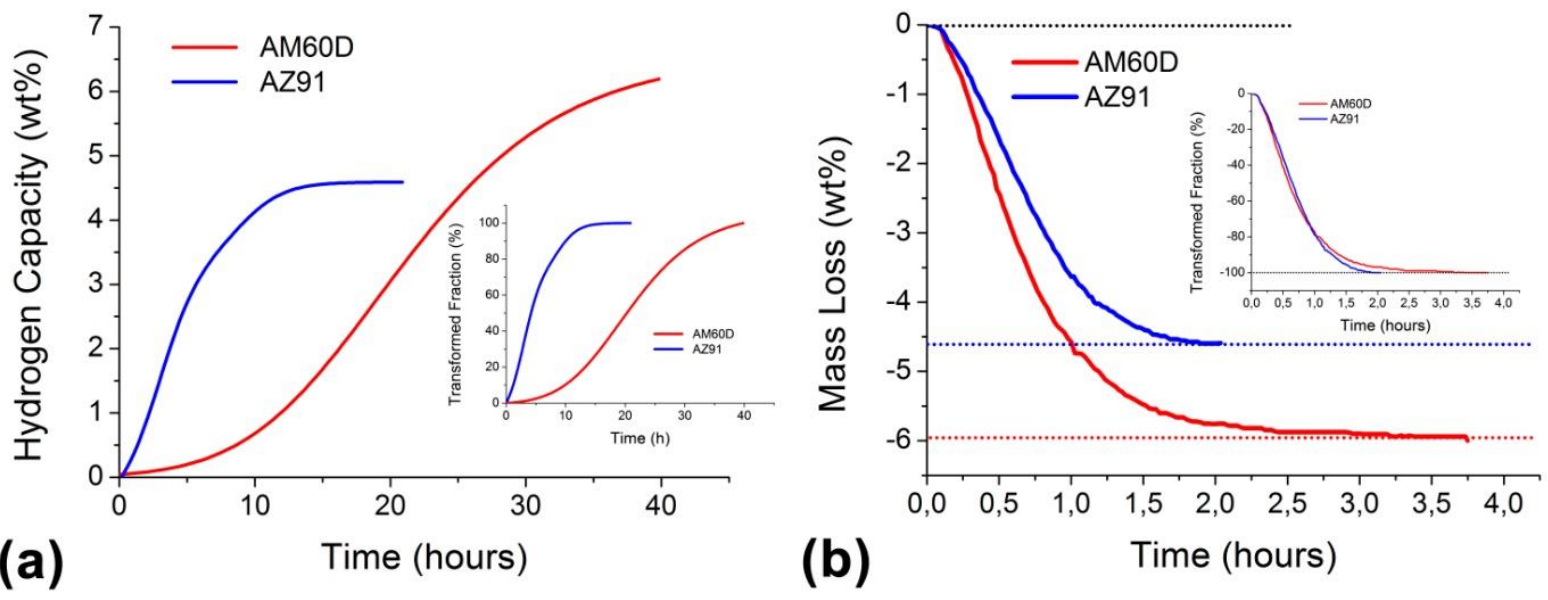

Figure 7 


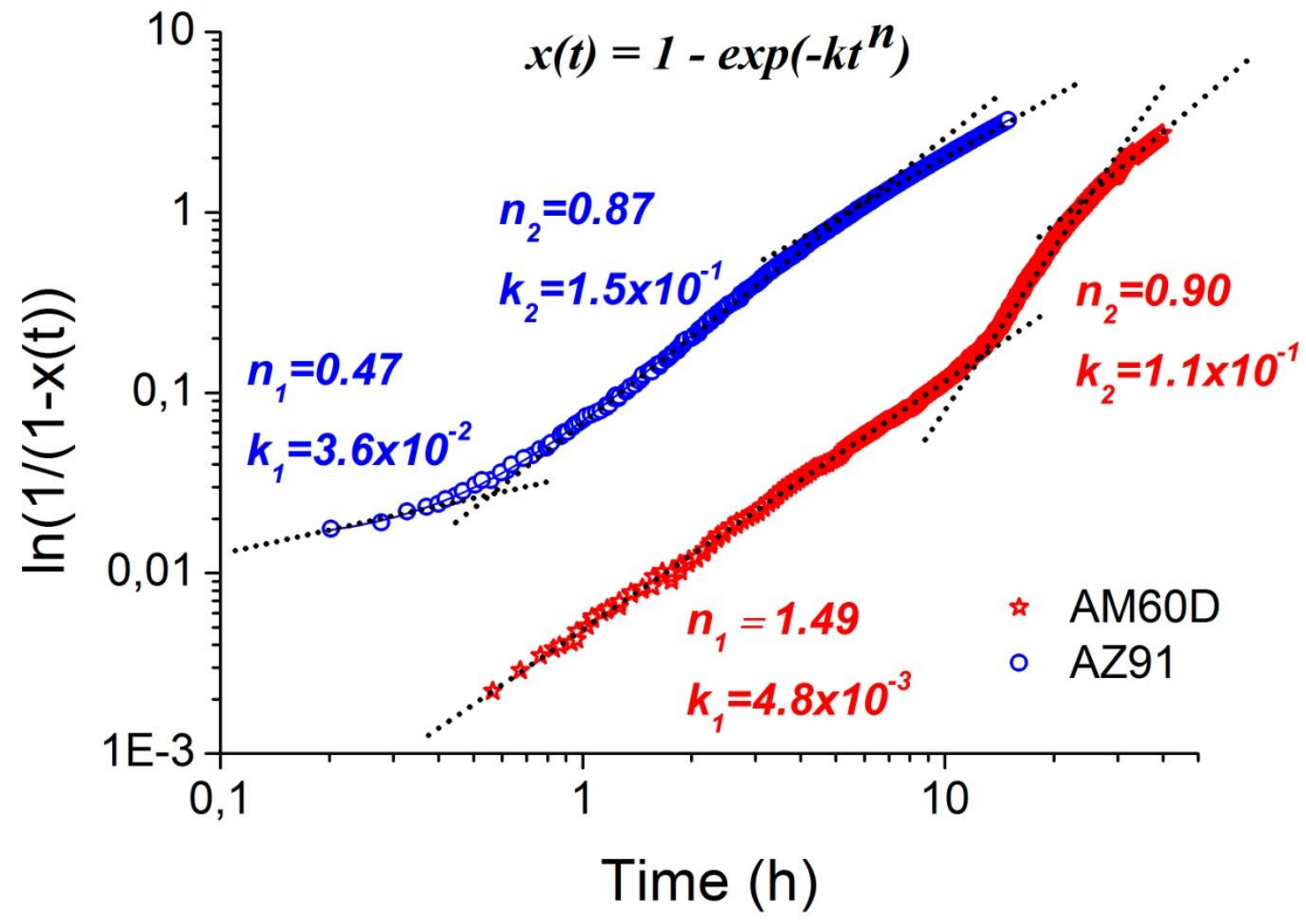

Figure 8 